\title{
Erich Kulke (1908-1997): Wandervogel, Volkskundler, Siedlungsplaner und VJL-Vorsitzender
}

\section{Eine erste Begegnung mit der Person}

Im Germanischen Nationalmuseum in Nürnberg befinden sich etwa 50 in den frühen 1960er-Jahren geschaffene Bauernhausmodelle aus dem deutschen Sprachraum sowie einige Pläne und Zeichnungen der "Forschungsstelle deutscher Bauernhof «, deren Leiter Erich Kulke war. ${ }^{1}$ Letztere sollten als Anregung für den Bau der Modelle dienen und gelangten in der Nachkriegszeit an das Museum. Ideengeschichtlich hängen beide Bestände zusammen. Informationen zur Forschungs- bzw. Mittelstelle und zur Hausforschung im Nationalsozialismus boten zunächst zwei Aufsätze des Volkskundlers und Hausforschers Klaus Freckmann, in denen Erich Kulke eine wichtige Rolle einnimmt. ${ }^{2}$ Erneut begegnete Kulke im Kontext der Ausstellung "Aufbruch der Jugend. Deutsche Jugendbewegung zwischen Selbstbestimmung und Verführung« im Jahr 2013. Im Ausstellungskatalog wurde seine Funktion als Vorsitzender des Vereins Jugendburg Ludwigstein zwischen 1959 und 1963 erwähnt, ${ }^{3}$ die wiederum der Grund ist, seine Person im Kontext der Geschichte auf der Burg im 20. Jahrhundert zu beleuchten.

Kulkes Verbindung zur Jugendbewegung knüpft aber auch an die Überlegungen Ernst Schlees (1910-1994) an, der 1966 schrieb »[...] für die Wissenschaftsgeschichte der Volkskunde wird sich früher oder später die Frage nach der Bedeutung der Jugendbewegung für das Ideengut des Faches stellen. $\aleph^{4}$

1 Die »Forschungsstelle deutscher Bauernhof» trug auch den Namen »Mittelstelle deutscher Bauernhof«; zu deren Aufgabe siehe weiter unten.

2 Klaus Freckmann: Hausforschung im Dritten Reich, in: Zeitschrift für Volkskunde, 1982, 78. Jg., S. 169-186. - Ders.: Zur Foto- und Plandokumentation in der Hausforschung der 30er und 40er Jahre, in: Zeitschrift für Volkskunde, 1985, 81. Jg., S. 40-50.

3 G. Ulrich Großmann: Jugendburgen, in: G. Ulrich Großmann, Claudia Selheim, Barbara Stambolis (Hg.): Aufbruch der Jugend. Deutsche Jugendbewegung zwischen Selbstbestimmung und Verführung (Ausstellungskatalog), Nürnberg 2013, S. 82-91, hier S. 85.

4 Ernst Schlee: Erich Meyer-Heisig zum Gedächtnis, in: Hessische Blätter für Volkskunde, 1966, 57. Jg., S. 253-255, hier S. 253. 
Blendeten Volkskundler, explizit Hausforscher, Kulkes Prägung in der Jugendbewegung aus, so schauten über die Jugendbewegung Forschende wenig auf seine Verortung in der Hausforschung. ${ }^{5}$ Der folgende Beitrag versucht, beide Bereiche zu beleuchten und auch Licht auf die Frage nach Kulkes Einstellung zum Nationalsozialismus zu werfen.

\section{Die bündischen Jahre bis 1933/35}

Erich Paul Otto Kulke wurde 1908 als Sohn des Baumeisters Paul Kulke und seiner Ehefrau in Frankfurt an der Oder geboren. Er besuchte das dortige evangelische Reformgymnasium, um danach eine Ausbildung zum Maurergesellen zu machen. 1927 führte ihn das Architekturstudium nach Karlsruhe, das er 1932 in Berlin bei Heinrich Tessenow (1876-1950) abschloss. Anschließend war er in seiner Heimatstadt als Regierungsbauführer und freier Architekt tätig. 1934 beendete er sein Studium mit der Dissertation »Die mittelalterlichen Burganlagen der Mittleren Ostmark «. ${ }^{6}$

Schon als Schüler wurde Kulke Mitglied und Führer des örtlichen Wandervogels. Zum zehnjährigen Bestehen der Gruppe gab die Zeitschrift »Wandervogel Deutscher Bund" 1932 das Themenheft "Frankfurt/Oder« heraus. Dem Vorwort Kulkes ist zu entnehmen, dass »ohne die Treue der Frankfurter Wandervögel« der Bund nicht entstanden wäre. "Ihr Durchhalten und ihre Opferbereitschaft $[\ldots]$ in allen Stunden geforderter Entscheidung schufen mir den Glauben an den Sinn und Sieg unseres Bundesweges. ${ }^{7}$ Der Weg war der, dass Kulke mit seiner Gruppe 1925 zum Wandervogel Völkischer Bund stieß. Dessen Bundesführer Karl Bückmann (1898-1946) verstand das Adjektiv »völkisch« im Sinne einer nicht-rassisch begründeten Volkstumsideologie. ${ }^{8}$ So schrieb er: »Wir glauben dabei mehr an die schöpferische Tat des Einzelwillens, der aus dem Bündnisleben Kraft und Antrieb gewinnt, als an eine geschlossene Massenwirkung des Bundes. Das ist der Sinn, den wir mit dem Begriff völkisch verbinden

5 Eine Ausnahme ist das Buch von Stefan Breuer, Ina Schmidt: Die Kommenden. Eine Zeitschrift der bündischen Jugend (1926-1933) (Edition Archiv der deutschen Jugendbewegung 15), Schwalbach/ Ts. 2010, S. 366-368.

6 Breuer, Schmidt: Kommenden (Anm. 5), S. 367.

7 Erich [Kulke]: Vorwort, in: Wandervogel Deutscher Bund (Osters), 1932, Folge 4, S. 91.

8 Karl Bückmann: Wandervogel, völkischer Bund (Ein Wort zur Aufklärung unseres vielfach mißverstandenen Namens), in: Werner Kindt (Hg.): Die deutsche Jugendbewegung 1920 bis 1933. Die bündische Zeit, Düsseldorf, Köln 1974, S. 246f. - Uwe Puschner: Völkische Bewegung und Jugendbewegung. Eine Problemskizze, in: Gideon Botsch, Josef Haverkamp (Hg.): Jugendbewegung, Antisemitismus und rechtsradikale Politik. Vom »Freideutschen Jugendtag« bis zur Gegenwart, Berlin u. a. 2014, S. 9-28. 
und $\mathrm{zu}$ einer Zeit verbunden haben, als man an eine parteimäßige völkische Richtung noch nicht dachte. ${ }^{9}$

1928 entstand dann der "Bund der Wandervögel und Kronacher «, als dessen Bundesführer Kulke im Juli 1929 auf Schloß Kleßheim bei Salzburg war, wo ein gemeinsamer Bundestag mit dem Österreichischen und dem Sudetendeutschen Wandervogel abgehalten wurde. ${ }^{10}$ Dort beschloss man das "Salzburger Bekenntnis«: »Wir [...] wissen, daß der Gedanke des Wandervogels weder überwunden noch erfüllt ist, und daß der Greif in allen deutschen Landen wieder zur Höhe steigt. Wir glauben an ihn als Erfüller unserer Jugendsehnsucht und Gestalter deutschen Lebens. Wir bekennen uns zu der Forderung, zuchtvoll an Leib und Seele unser Leben für Volk und Heimat führen zu wollen. " ${ }^{11}$ Abschließend kritisierte Kulke die Meißnerformel, da sie als ein geschichtliches Ereignis ohne Wirkung geblieben sei. Vielmehr rühmte er das "Langemarck-Geschlecht «, das ebenso wie die 600 in Salzburg versammelten "deutschbewußten« Jungen und Mädchen bereit gewesen war, seine "Lebenskraft in Verbundenheit und Verantwortung dem Boden des Vaterlandes zu opfern. " $^{12}$ Mit dem "LangemarckGeschlecht « nahm Kulke Bezug auf die jugendlichen Opfer des Stellungskrieges in Flandern im November 1914, unter denen rund 10.000 Wandervögel waren. Die Niederlage wurde in der Kriegserzählung in einen Sieg umgedeutet, wonach die Jugendlichen nicht umsonst gefallen seien. ${ }^{13}$

Die Generationenunterschiede im »Bund der Wandervögel und Kronacher» führten im Januar 1930 in Kassel zu einer Abspaltung. Die Jüngeren mit Erich Kulke an der Spitze vermissten »eine klare Einstellung der Bundesführung als Bekenntnis für Volk und Volkstum. « ${ }^{14}$ Man gründete den »radikal-völkischen « ${ }^{15}$ Wandervogel Deutscher Bund mit Kulke als Führer. Das Bundesmotto lautete: »Wandervogel als Grundlage, Volk als Aufgabe ${ }^{16}{ }^{16}$ Schwergewicht der Arbeit

9 Bückmann: Wandervogel (Anm. 8), S. 246.

10 Kindt: Jugendbewegung (Anm. 8), S. 1266.

11 Erich Kulke: Das Salzburger Bekenntnis, in: Die Kommenden. Großdeutsche Wochenschrift aus dem Geiste volksbewußter Jugend, 1929, Nr. 4, 36. Folge, S. 426 (mit Kommentar). - Mit leicht verändertem Wortlaut ist das Salzburger Bekenntnis wieder abgedruckt in: Wandervogel Deutscher Bund, 1932, Nr. 3, S. 62.

12 Ebd.

13 Gert Krumeich: Langemarck, in: Etienne François, Hagen Schulze (Hg.): Deutsche Erinnerungsorte, 3. Bd., München 2001, S. 292-309. - Arndt Weinrich: Der Weltkrieg als Erzieher. Jugend zwischen Weimarer Republik und Nationalsozialismus, Essen 2013.

14 Erich Kulke: Der Weg unseres Bundes, in: Fünfzig Jahre Wandervogel deutscher Bund. Wege und Aufgaben einer bündischen Gemeinschaft. Bussau 1979, S. 2-12, hier S. 3.

15 Winfried Mogge: »Wir lieben Balder, den Lichten...« Völkisch-religiöse Jugendbünde vom Wilhelminischen Reich zum »Dritten Reich«, in: Uwe Puschner, Clemens Vollnhals (Hg.): Die völkisch-religiöse Bewegung im Nationalsozialismus. Eine Beziehungs- und Konfliktgeschichte, Göttingen 2012, S. 45-64, hier S. 51.

16 Kulke: Weg (Anm. 14), S. 4. 
sollte die »Einsatzbereitschaft für das von Gefahren bedrohte Volk durch Lied, Tanz, Laienspiel, Volksmusik und Grenzlandarbeit« bilden. ${ }^{17}$ Volkslied und - $\tan z$ betrachtete Kulke als wahren Lebensausdruck, »freche und geile Gassenhauer« hingegen als "fremden Geist«, den er auch bei jüdischen Künstlern und Schriftstellern anprangerte und somit können an seiner Person Tendenzen des völkischen Antisemitismus festgemacht werden. ${ }^{18}$

1930 kam es auf der Veste Coburg zum "Bündnis der Greifen und Falken« unter der Schirmherrschaft des völkisch orientierten Schriftstellers Wilhelm Kotzde-Kottenrodt (1878-1948). Es unterzeichneten Fritz Lang als Führer des Österreichischen Wandervogels, ${ }^{19}$ Erich Kulke für den Wandervogel Deutscher Bund sowie Karl Dietrich (1899-1983) für die Deutsche Falkenschaft und den Schwarzhäuser Ring. ${ }^{20}$ Unter Beibehaltung ihrer Selbständigkeit wollten die Bünde »von der kulturellen Seite her mithelfen am Bau des kommenden großdeutschen Reiches «. ${ }^{21}$ Große Aufgaben sah man vor allem in Ostmitteleuropa, wo man die dort lebenden Deutschen durch die oben skizzierte Kulturarbeit stärken wollte. 1931 schrieb Kulke: »Wenngleich die Ostfrage vielleicht im Augenblick die brennendste Not des deutschen Volkes aufrollt, so wird doch das Schicksal unserer gesamten Volkseinheit nicht nur im Osten entschieden. Was wir ersehnen [...], das bleibt ein Großdeutschland mit Einschlusse all derer, die durch den Versailler Vertrag vom Mutterland getrennt wurden. $\aleph^{22}$ Kulke und sein Bund verfolgten also durchaus revisionistische Ziele, auch wenn die Mittel der »Grenzlandarbeit" scheinbar harmlos waren, wie die Versendung einer bedeutenden Zahl von Büchern in den Osten. ${ }^{23}$ Die völkische Ausrichtung des Akteurs offenbart sich in seiner expliziten Werbung für die Zeitschrift "Die Kommenden " in einem Führerbrief 1930 ebenso wie in einem an seinen Bund gerichteten Artikel 1931: „Wir Älteren aber wollen den Wandervogel [...] begreifen lernen, als eine Befreiungsbestrebung des alten deutschen Adelsblutes, unter dessen Führung allein unser Volk als menschheitsnötiges erhalten bleiben

17 Kindt: Jugendbewegung (Anm. 8), S. 249-250, hier S. 250.

18 Erich Kulke: Dem »Wandervogel Deutscher Bund«, in: Die Kommenden, 1931, Nr. 6, Folge 28, S. 326. - Dazu auch Peter Ulrich Hein: Völkische Kunstkritik, in: Uwe Puschner, Walter Schmitz, Justus H. Ulbricht (Hg.): Handbuch zur Völkischen Bewegung 1871-1918, München u. a. 1996, S. 613-633 sowie Werner Bergmann: Völkischer Antisemitismus im Kaiserreich, ebd., S. 449-463.

19 Fritz Lang war mit seiner Frau auch beim Treffen zum Jubiläum "50 Jahre Wandervogel Deutscher Bund« auf Burg Ludwigstein 1979; vgl. Fünfzig Jahre Wandervogel deutscher Bund (Anm. 14), S. 18.

$20 \mathrm{Zu}$ Karl August Gottlob Dietrich: Breuer, Schmidt: Kommenden (Anm. 5), S. $321 \mathrm{f}$.

21 Kindt: Jugendbewegung (Anm. 8), S. 867.

22 Aelterenbrief (AdJb, Z/100-1013).

23 Erich Kulke: Bundeskapiteltagung 1931 anlässlich des Kronacher Bundestages, in: FuehrerBriefe, hg. vom Wandervogel Deutscher Bund, Hartung [Januar] 1931, Folge 6 (AdJb, Z/ 100-1477). 
und wieder hochkommen kann. ${ }^{24}$ Und auch 1932 nannte er als »Hochziel« des Bundes die Erziehung zum "Herrenmenschen ${ }^{25}$, was wiederum dessen elitären Anspruch verdeutlicht.

Kulke erinnerte sich 1979 an die Jahre ab 1933 und beschrieb sie als »stürmische Zeit» und als ein »Ringen um das Weiterleben als Bund bis 1935/37«. Dem «massiven Druck der Hitlerjugend, der Reichsjugendführung« suchte er durch »Vorsprachen und Beschwerden beim gleichgeschalteten Reichsbund der Jugendverbände« zu begegnen entgegnen, doch nutzten diese nichts, und so gab er in »einem Rundbrief die Weisung, den Bund zur Sommersonnenwende 1933 aufzulösen. ${ }^{26}$ Zuvor, im Januar 1933, hatte sich Kulke in einen Führerbrief an seinen Bund gerichtet, in dem er diesem einen Weg in die Zukunft weisen wollte: »Jeder Gau unter klarer Führung strebe danach, im nächsten Jahre eine weit stärkere Selbständigkeit im Vertrauen auf seine ihm selbst gestellten Aufgaben zu erzielen. ${ }^{27}$ Diese Aufgaben sah er vor allem in kultureller Hinsicht, doch als "Voraussetzung jeder Kultur» betrachtete er die "Wehrhaftigkeit» und den »Willen zur Wehrhoheit unseres Volkes«. Ferner hieß es: „Der Kampf um die Kultur [...] fordert das Bekenntnis zum Krieger. Wir prägen den Begriff des wehrhaften Bauernstandes, als Ausdruck der Schollenverbundenheit und des Wehrwillens unseres Bundes. « Die Arbeit an der»Waffenreife» sollte ein zweites Langemarck verhindern. Im Februar appellierte er an die "Jungen und Mädel« des Bundes, Landarbeit für die Artamanen zu leisten. ${ }^{28}$ Der Führerbrief aus dem März 1933 trug das Thema "Luftschutz. Gas und Bomben drohen«. Kulke begründete ihn mit der "völligen Ohnmacht» der deutschen Wehrhaftigkeit nach dem Versailler Vertrag und listete Möglichkeiten des Luftschutzes auf. Letztlich stachelte er aber auch die Aggression an: »In einer Stunde können feindliche Geschwader von allen Seiten jede deutsche Stadt von der Grenze aus erreichen! ${ }^{29}$ Seit dem 15. März 1933 war Kulke selbst Mitglied der Hitlerjugend und hatte den Rang eines Scharführers. ${ }^{30}$ Der Wandervogel Deutscher Bund sollte »in

24 Erich Kulke: Zum neuen Bund, in: Die Kommenden, 1931, Nr. 6, Folge 28, S. 331. - Ähnlich auch ders.: Wandervogel, Deutscher Bund, in: Wandervogel Deutscher Bund, 1931, Folge 1/ 2, S. 4-6, hier S. 5.

25 Erich Kulke: Thing. Worte zum Bundesthing, in: Wandervogel Deutscher Bund, 1932, Folge 10/11, S. 225-227, hier S. 225.

26 Kindt: Jugendbewegung (Anm. 8), S. 229-250. - Kulke: Weg (Anm. 14), S. 5-6.

27 Erich Kulke: An den Bund!, in: Fuehrer-Briefe, hg. vom Wandervogel Deutscher Bund, Hartung [Januar] 1933, Folge 1, Blatt 2 (AdJb, Z /100-1477).

28 Fuehrer-Briefe, hg. vom Wandervogel Deutscher Bund, Hornung [Februar] 1933, Folge 2 (AdJb, Z /100-1477).

29 Fuehrer-Briefe, hg. vom Wandervogel Deutscher Bund, Lenzmond [März] 1933, Folge 3 (AdJb, Z /100-1477).

30 Openjur VG Frankfurt (Oder) - Urteil vom 16. Oktober 2008 - Az. 4 K 1114/06, Satz 8, Zugriff 09.01.2013. Den Hinweis auf diese Quelle verdanke ich Klaus Freckmann. - Nach eigenen Angaben war Kulke schon seit 1932 in der Hitlerjugend. Vgl. Bundesarchiv Berlin 
seiner Organisation« aufgelöst und heimlich weitergeführt werden. So existierte die Älterenorganisation ohne die Jüngeren im Rahmen des »Kampfbundes für Deutsche Kultur« und des Deutschen Wandervogels beim »Fachamt für Bergsteigen und Wandern« im »Reichsbund für Leibesübungen« fort, an dessen Spitze Karl Bückmann stand.

Im August 1933 betrachtete Kulke die Position des Bundes zur NSDAP als "geklärt«, da »ein wesentlicher Teil unserer Aelteren an führender Stelle innerhalb der Partei ihr Betätigungsfeld gefunden hat. Wir bejahen die Geschehnisse der letzten Zeit als einen Beginn zur Neugestaltung des deutschen Lebens überhaupt. Zum Führer Adolf Hitler haben wir nach seinem bisherigen Verhalten, das so wenig diktatorischen Anstrich gewonnen hat, Vertrauen zur Gestaltung des deutschen Freiheitsrechtes. ${ }^{31}$ Der Bund bekannte sich begeistert zur »Totalität der deutschen Revolution«, die für ihn »ein immerwährendes Ringen um die Tiefe der deutschen Weltanschauung « war. ${ }^{32}$ Die "völkische Bewegung " konnte demnach nur erfolgreich sein, wenn sie sich Eingang in die "religiösen Machtbereiche des deutschen Wesens« verschaffe, man war auf der Suche nach einer »deutschen Weltanschauung $"{ }^{33}$ Auch in Kulkes Beitrag »Wille und Glaube« ging es um das »Streben nach dem deutschen Sinnbild « und einem "arteigenem Glauben «. ${ }^{34}$ So stand er auch der Deutschen Glaubensbewegung nah. ${ }^{35}$

Besonders stürmisch gestalteten sich die Jahre ab 1934 für Kulke, der aber 1979 angeblich nur noch "verblasste Erinnerungen" an die Zeit hatte, die er anhand der Akten zwar auffrischen konnte, allerdings nicht ausführlich behandeln wollte und an andere Personen verwies. ${ }^{36}$ Zum Jahreswechsel 1934/35 schrieb er einen Brief an die Österreichischen Wandervögel und die »Kameraden vom alten Bund $"{ }^{37}$ in dem er den nationalsozialistischen Staat begrüßte: »Wir im Reich stehen schon längst gestaltend und führend in der aufgabenreichen Gegenwart und wollen Tag und Nacht mit dem Einsatz der besten Kräfte uns hineinwerfen in den Strom deutschen Lebens, der sich uns wie ein unfaßbares Wunder täglich neu offenbart.« Den Österreichern sprach er Mut zu im »Kampf um ein nationalsozialistisches freies Oesterreich «.

(BArch), Antrag zur Bearbeitung der Aufnahme als Mitglied der Reichsschriftumskammer, Gruppe Schriftsteller vom Juli 1941.

31 Erich [Kulke]: Zur Lage, in: Wandervogel Warte. Zeitschrift der Volkschaft des Wandervogels Deutscher Bund, 1933, Folge 4, S. 134f., hier S. 134.

32 Ebd.

33 Ebd.

34 Erich [Kulke]: Wille und Glauben, in: Wandervogel Warte. Zeitschrift der Volkschaft des Wandervogels Deutscher Bund, 1933, Folge 6, S. $140 \mathrm{f}$.

35 Breuer, Schmidt: Kommenden (Anm. 5), S. 125.

36 Kulke: Weg (Anm. 14), S. 5.

37 AdJb, A 187 Nr. 21, 3: WVDB 1933-1942. 
Am 13. Januar 1935 richtete sich Kulke an Karl Bückmann und Wolf Wieckberg (1894-1986) und erklärte seinen Rücktritt als Führer, "weil es allein Aufgabe des Bundesführers oder seines Vertreters ist, die Weite der Bundesausrichtung zu bestimmen. ${ }^{38}$ Weiter hieß es: „Wir haben nichts Grösseres zu kennen, als in Eindeutigkeit für das Reich unseres Führers tätig zu sein. Einsatz in den Organisationen jeglicher Art bleibt oberste Forderung."Kulke vertrat die Ansicht, die Ausrichtung des Bundes habe nach folgenden Gesichtspunkten zu erfolgen:

»Der politische, deutsche Mensch

Der romfreie, deutsche Mensch

Der im Volkstum verwurzelte, deutsche Mensch»

Bückmann wandte sich am 19. Januar 1935 "an alle im Wandervogel« und schloss Kulke aus dem Deutschen Wandervogel aus, weil er nicht versucht hätte, die ihm übertragene Aufgabe als Führer der Jungmannschaft zu erfüllen. Ferner nahm er Stellung zu den von Kulke aufgeführten drei Punkten. ${ }^{39}$ Zum ersten Punkt schrieb er, der bereits vor 1933 Landtagsabgeordneter der NSDAP war, u. a.: »Ich lasse mich [sic!] hierbei von keinem, der nach der Machtübernahme zur NSDAP gestossen ist, irgendwelche Vorschriften machen, [...] Wer in den Kampfjahren zur Mitarbeit nicht bereit war, sollte heute nicht allzu laut von seinem politischen Einsatz sprechen." Hinsichtlich des zweiten Punktes erinnerte Bückmann an seinen Aufruf in Questenberg, "gegen den Totalitätsanspruch der Kirche Front zu machen... « und Kulkes Forderung im letzten Punkt erachtete er als reine Phrase. Er schloss den Brief mit dem Fazit: „Erich Kulkes Versuch, einen kleinen Wandervogelbund in die Front der Großorganisationen zu stellen, verfällt nur dem Fluch der Lächerlichkeit. « Erich Kulke schrieb darauf am 28. Januar einen Brief an seine Kameraden, in dem er über seinen Ausschluss aus dem Deutschen Wandervogel informierte und der Bundesführung riet, den Bund aufzulösen. ${ }^{40}$ Seiner Meinung nach wurde das Wort Bund missbraucht, »wenn nicht Angriffsgeist, Opfersinn und Zielsicherheit dahinterstecken." Zudem ließ er wissen, dass er keinen neuen Bund innerhalb der NS-Organisationen aufziehen wollte. Später, wohl kurz nach seinem Ausschluss durch Bückmann, ergab sich die Möglichkeit einer Weiterarbeit mit seinen Gefolgsleuten an dem im April 1933 gegründeten »Reichsbund Volkstum und Heimat« unter Leitung von Werner Haverbeck (1909-1999). Dieser kam aus der evangelischen Jugendbewegung und war schon in den 1920er-Jahren in Organisa-

38 Ebd.

39 Ebd.

40 Ebd. 
tionen der NSDAP und seit 1929 Parteimitglied. ${ }^{41}$ Ab 1931 leitete er das Amt für Kultur und Weltanschauliche Erziehung in der Reichsjugendführung, wurde aber, weil er gegen die paramilitärische Ausbildung der Jugend durch Baldur von Schirach war, 1932 all seiner Ämter enthoben. 1933 folgte im Reichsbund ein Comeback für ihn, das schon 1934 endete, ${ }^{42}$ weil er auf die Jugend und die Arbeiterschaft setzte und so die Heimatverbände verprellte. ${ }^{43}$ Kulke und seine Kameraden stießen offenbar erst nach dem Ausscheiden Haverbecks zum Reichsbund.

Während der Olympischen Spiele 1936 kam es laut Kulke zu einem letzten Treffen der »alten bündischen Freunde« in Steinhöfel bei Fürstenwalde im Rahmen des »Reichsbundes Volkstum und Heimat $~^{44}$ Für ihn folgte nach eigenen, bisher nicht nachweisbaren Angaben eine scharfe Verwarnung mit Überwachung durch den Sicherheitsdienst, der eine »eigene >Akte Kulke«" führte, wie er durch einen ihm »nahestehenden befreundeten SS-Führer erfuhr . $^{45}$

Aus dem bisher Erwähnten geht also hervor, dass Kulke kein Parteimitglied, aber Mitglied der Hitlerjugend war und offenbar nach der Machtergreifung die Nähe zur NSDAP suchte. ${ }^{46}$ Dass er sich aktiv für den Nationalsozialismus einsetzte, zeigt auch die von ihm herausgegebene Publikation "Hin zu dem Tag der Deutschen Freiheit! Sinnsprüche für Arbeit und Feier«. Im Vorwort zur dritten Auflage erwähnt Kulke die zwei vergriffenen Auflagen des Bändchens aus dem Jahr 1934. Danach hatte es im ganzen Reich Beachtung gefunden und »die ständig wachsende Nachfrage" hatte Veranlassung zu einer Neuauflage im Herbst 1935 gegeben, die unter der Losung "Sinnsprüche der Hitler-Jugend für Alltag und Feier» erschien. Sie war gedacht »als Gabe der Hitler-Jugend an alle NS.-Formationen, ganz gleich, in welcher Kolonne sie shin zu dem Tag der

41 Kulke: Weg (Anm. 14), S. 6. - Kulke verwies hier auf die Präsidentschaft Haverbecks der Sektion Deutschland im »Weltbund zum Schutz des Lebens«. Hierzu Ingrid Tomkowiak: Das "Heidelberger Manifest « und die Volkskunde, in: Zeitschrift für Volkskunde, 1996, 92. Jg., S. 185-207, bes. S. 196-199, hier S. 197. Sie wies auf die Reaktivierung einer völkisch-mythischen Ideologie und die Modernisierung der Volkstumsideologie durch Haverbeck in den 1970er- und 1980er-Jahren hin.

42 Zur vielschichtigen Persönlichkeit von Werner Haverbeck siehe u. a. Hermann Bausinger: Volkskunde und Volkstumsarbeit im Nationalsozialismus, in: Helge Gerndt (Hg.): Volkskunde und Nationalsozialismus. Referate und Diskussionen einer Tagung, München 1987, S. 131-141. - Tomkowiak: Manifest (Anm. 41), bes. S. 196-199.

43 Breuer, Schmidt: Kommenden (Anm. 5), S. 128.

44 Kulke: Weg (Anm. 14), S. 6. - Den Reichsbund löste Robert Ley allerdings schon im Januar 1935 auf.

45 Kulke: Weg (Anm. 14), S. 6.

46 Die letzte Angabe müsste überprüft werden, würde aber den Rahmen dieser Studie sprengen. Im Bundesarchiv Berlin liegen in der Personalakte Kulke zumindest keine entsprechenden Hinweise vor. 
Freiheit marschieren. ${ }^{47}$ Eine vierte Auflage des Werkes mit einer Auflage von 4.500 Stück erschien $1940 .^{48}$ Die in dem Buch wiedergegebenen Zitate verweisen teilweise auf die frühere publizistische Tätigkeit Kulkes, denn auch in den bündischen, zum Teil von ihm selbst herausgebrachten Zeitschriften finden sich gelegentlich entsprechende Zitate. Die Bandbreite reicht von Goethe über Hermann Löns, Ernst Moritz Arndt, Georg Stammler, Walter Flex, Ernst Jünger, Alfred Rosenberg bis hin zu Adolf Hitler. Ein Gericht stufte das Bändchen 2008 eindeutig als nationalsozialistische Propaganda ein. ${ }^{49}$

\section{Kulkes Weg nach 1935}

1935 heiratete Kulke Ilse Prassen (1908-2000) im Zisterzienserkloster Chorin. Sie war Mitglied des Österreichischen Wandervogels und hatte 1931 in den »Kommenden« einen Aufsatz über die »Mädelgrenzarbeit in Slavonien« veröffentlicht ${ }^{50}$ in dem sie über die praktische Arbeit vor Ort und die verschiedenen Maßnahmen wie gemeinsames Lesen deutscher Bücher und Säuglingspflege berichtet. Die Wandervögel wollten auf diese Weise Auslandsdeutsche zur Selbständigkeit in der Erhaltung und Pflege des "Deutschtums « erziehen. ${ }^{51}$ Mithin ergänzte sich das Paar in seinen Interessen. Es zog in die Nähe von Berlin und bekam im Laufe der Jahre sieben Söhne. Seit 1936 war Kulke im Stabsamt des Reichsbauernführers für Bau- und Siedlungsfragen zuständig. ${ }^{52}$ Im Sommer

47 Erich Kulke (Hg.): Hin zu dem Tag der deutschen Freiheit! Sinnsprüche für Arbeit und Feier, Berlin 1935.

48 Openjur (Anm. 30), Satz 10.

49 Ebd., Satz 60.

50 Ilse Krassen [Prassen; C.S:]: Mädelgrenzarbeit in Slavonien, in: Die Kommenden, 1931, 6. Jg., 36. Folge, S. 424-426.

51 Breuer, Schmidt: Kommenden (Anm. 5), S. 98, 181. Aufgrund eines Druckfehlers in der Zeitschrift wurde in diesem Band der Nachname irrigerweise als Krassen wiedergegeben. Ferner findet sich der Holzschnitt "Unser Sternsingen" von ihr in der Zeitschrift des Wandervogel Deutscher Bund, 1931, Folge 5, S. 87-89.

52 Erich Kulke: Die Vorlauben des Oderranddorfes Zäckerick, in: Adolf Spamer: Märkisches Volkstum. Brandenburgische Jahrbücher, 1936, Nr. 3, S. 224-232, hier S. 224. - Mit kleinen Abweichungen und anderem Bildmaterial wieder abgedruckt: Erich Kulke: Die Laubenhäuser des Oderranddorfes Zäckerick (Aus der bevorstehenden Veröffentlichung: "Der Bauernhof im Gebiet der unteren Oder«.), in: ders. (Bearb.): Vom deutschen Bauernhof. Vorträge der ersten Arbeitstagung der Arbeitsgemeinschaft für Deutsche Volkskunde. München 1939, S. 142-153. Hiernach fanden die Untersuchungen erst im September 1937 statt, was aber im Hinblick auf das Erscheinungsdatum der Brandenburgischen Jahrbücher unwahrscheinlich erscheint. - Dazu auch: Leonore Scholze-Irrlitz: Feldforschung in der Mark Brandenburg. Volkskundliche Wissensproduktion in den 1930er Jahren in Berlin, in: Ina Dietzsch, Wolfgang Kaschuba, Leonore Scholze-Irrlitz (Hg.): Horizonte ethnografischen Wissens. Eine Bestandsaufnahme, Köln u. a. 2009, S. 112-130, bes. S. 115. 
des Jahres unterrichtete er Volkskunde an der Hochschule für Lehrerbildung. ${ }^{53}$ Am 1. Mai 1937 trat er schließlich in die NSDAP ein, was ihm vorher wohl aufgrund der Mitgliederaufnahmesperre vom 19. April 1933 nicht möglich war. Nun konnten diejenigen der Partei beitreten, die sich inzwischen in NS-Verbänden und NS-Organisationen als Nationalsozialisten bewährt hatten. Zwischen 1939 und 1941 war Kulke Pionieroffizier, von 1942 bis 1944 Architekt in der Siedlungsabteilung des Oberkommandos des Heeres und dann an der Front. 1943 habilitierte er sich an der Technischen Hochschule in Danzig mit der Arbeit »Die Laube als ostgermanisches Baudenkmal «. ${ }^{54}$

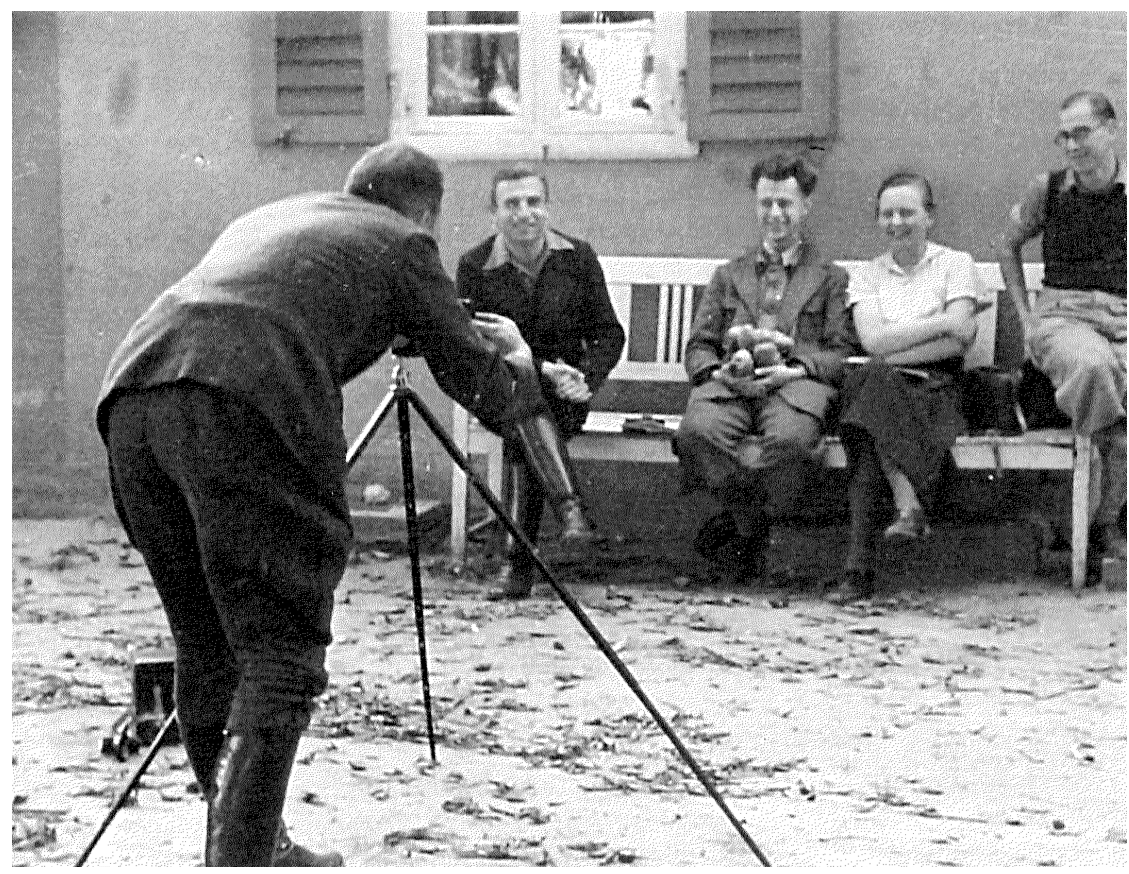

Abb. 20: Seit 1936 war Kulke im Stabsamt des Reichsbauernführers für Bau- und Siedlungsfragen zuständig.

Als im Januar 1937 die »Arbeitsgemeinschaft» beziehungsweise »Reichsarbeitsgemeinschaft für deutsche Volkskunde« gegründet wurde, gehörten zu ihren Trägern die Reichsleiter der NSDAP Richard Walther Darré, Heinrich Himmler und Baldur von Schirach; ihr Leiter war Alfred Rosenberg. Die Arbeitsgemeinschaft sollte eine Beratungs- und Mittelstelle sein, deren Ergebnisse allen Parteigliederungen zugänglich waren. Die Volkskunde betrachtete man

53 Openjur (Anm. 30), Satz 12.

54 Breuer, Schmidt: Kommenden (Anm. 5), S. 367. 
damals "als das Kerngebiet jeglicher Erziehungs- und Schulungsarbeit «. ${ }^{55}$ Dementsprechend sollte diese noch junge Wissenschaft »zu einem Bollwerk der nationalsozialistischen Weltanschauung " ausgebaut werden. ${ }^{56}$ Erich Kulke leitete die »Mittelstelle für deutsche Bauernhausforschung«. Zugleich war er politischer Leiter in der "Hauptstelle Volkskunde» in Matthes Zieglers Amt »Weltanschauliche Informationen«. Ziegler (1911-1992), seit 1931 NSDAPMitglied, und Kulke kannten sich möglicherweise aus ihrer bündischen Zeit, denn der Nürnberger war seit 1929 Mitglied der Adler und Falken. ${ }^{57}$

Im März 1938 referierte Kulke über »Die Arbeitsgrundlagen der >Mittelstelle deutscher Bauernhof «. Es war die Aufgabe dieser Einrichtung, die Fragen der Hofgestaltung und des Hausbaues zu untersuchen, die »für die Erkenntnis einer rassengeschichtlichen und rassenbedingten Volkskunde von Wert sind. ${ }^{58}$ Als Arbeitsgebiete der Mittelstelle im Bereich von Forschung und Pflege führte er an:

1. Entwicklungsgeschichte des germanischen Bauernhofes vom Nordischen, nicht vom römisch $=$ südländischen Standpunkt aus. ${ }^{59}$

55 Hannjost Lixfeld: Aufbau und Aufgaben Rosenbergs Reichsarbeitsgemeinschaft für deutsche Volkskunde, in: Wolfgang Jacobeit, Hannjost Lixfeld und Olaf Bockhorn (Hg.): Völkische Wissenschaft. Gestalten und Tendenzen der deutschen und österreichischen Volkskunde in der ersten Hälfte des 20. Jahrhunderts, Wien u. a. 1994, S. 205-217, hier S. 207.

56 Zit. nach Lixfeld: Aufbau (Anm. 55), S. 208.

57 Lixfeld: Aufbau (Anm. 55), S. 207. - Zu Ziegler und dem »Amt Rosenberg« auch: Esther Gajek: »Feiergestaltung«. Zur planmäßigen Entwicklung eines »aus nationalsozialistischer Weltanschauung geborenen, neuen arteigenen Brauchtums" am "Amt Rosenberg", in: Bayerisches Jahrbuch für Volkskunde, 2000, S. 75-86, bes. S. 76f. - Manfred Gailus: Vom "gottgläubigen« Kirchenkämpfer Rosenbergs zum »christgläubigen« Pfarrer Niemöllers: Matthes Zieglers wunderbare Wandlungen im 20. Jahrhundert, in: Zeitschrift für Geschichtswissenschaft, 2006, 54. Jg., S. 937-973.

58 Erich Kulke: Die Arbeitsgrundlagen der "Mittelstelle deutscher Bauernhof" (Auszug aus dem am 17. März 1938 anlässlich der Bauernhofforschertagung gehaltenen Vortrag), in: Kulke: Bauernhof (Anm. 52), S. 17-26, hier S. 18.

59 Diesen Arbeitspunkt deckte Kulke z. B. ab in den Beiträgen: Der »keltische« Holzbaustil am oberdeutschen Bauernhaus, in: Germanen-Erbe. Monatsschrift für Deutsche Vorgeschichte. Amtliches Organ des Reichsbundes für Deutsche Vorgeschichte und der Hauptstelle Vorgeschichte des Beauftragten des Führers für die gesamte geistige und weltanschauliche Schulung und Erziehung der NSDAP, 1938, Nr. 3, S. 368-374. (Exemplarisch S. 368: "Jede kulturelle Erscheinung auf germanischem Raume gilt solange als diesem germanischen Volksboden entwachsen, als nicht das Gegenteil zwingend erwiesen ist. Dieser Grundsatz erscheint uns heute ganz natürlich, für jeden Deutschen selbstverständlich; und doch ist es noch gar nicht so lange her, daß eine ganze Anzahl deutscher Forscher und Gelehrter immer wieder ihre geradezu krankhafte Neigung bekundete, jede kulturelle Hochleistung, ohne jegliche wissenschaftliche Begründung, lieber irgendwelchem Fremdvolk, als dem eigenen Volke zuzuschreiben.«) - Die Entwicklung des nordischen Bauernhauses und sein Einfluß auf die nordisch bestimmte Kultur, in: Odal. Monatszeitschrift für Blut und Boden, 1936, Nr. 4, S. 1-19. 
2. Herausbildung des Bauernhof=Begriffes im Gegensatz und zur Ergänzung der bisher betriebenen einseitigen Hausforschung. [...]

3. Erweiterung der bisher gewonnenen Ergebnisse der germanisch-deutschen Landnahme und der damit verbundenen deutschen Siedlungsgeschichte.

4. Pflege alter wertvoller Bauerngehöfte im Sinne des staatlichen Denkmalsschutzes, da das Bauerngehöft uns einen mindestens genau so bedeutsamen nationalen Gemeinsitz darstellt, wie Klöster, Schlösser, Kirchen und Burgen.

5. Reinigung und Erneuerung der bäuerlichen Bauweisen. [...]

Über die Arbeit der Mittelstelle berichtete Kulke auch 1939 in den »Nationalsozialistischen Monatsheften.$^{60}$ Hier prangert er vor allem den Niedergang der Baukunst seit der Mitte des 19. Jahrhunderts an, die auf dem Dorf zu einer »blutleeren Gestaltung" geführt habe und für die Nachkriegsjahre konstatierte er den »Irrwahn eines internationalen Baustiles $",{ }^{61}$ eine deutliche Positionierung gegen die Architekten des Bauhauses. »Dachpappe, Zement und Wellblech, eine in den Dörfern übel verteilte Geschäftsreklame für Zigaretten und Nähmaschinen taten dazu ihr übriges, um das einheitliche Dorfbild auf eine kulturell möglichst niedrige Stufe herabzudrücken. ${ }^{62}$ Um einer derartigen $»$ Dorfverschandelung" entgegen zu wirken, die in seinen Augen zur "Auflösung der bäuerlichen Lebenssicherheit« führte und die damalige Staatsideologie gefährdete, zeigte er Möglichkeiten "zur Erhaltung und Pflege der landschaftsgebunden Bauweisen « auf. ${ }^{63}$ Ein Punkt war »Die Erziehung der bäuerlichen Bauherrn, der ländlichen Plangestalter und des fachlichen Nachwuchses $« .^{64}$ Dabei schlug er für Hochschulstudenten in den Dörfern "regelrechte etwa einwöchige Lager" vor, ${ }^{65}$ eine Veranstaltungsform, die auch in der Jugendbewegung in den 1920erJahren an Popularität gewonnen hatte. Neben einigen anderen Aufgaben wollte er die Deutsche Arbeitsfront sowie den Reichsnährstand mit der »Beseitigung der häßlichen Reklame« betrauen. Die "Dorfverschönerung« bzw. die "Volkstumspflege« in Form der Baupflege sollte letztlich auch der Landflucht Einhalt gebieten. ${ }^{66}$ Das "schöne Dorf ${ }^{67}$ oder das "heimatgebundene Bauen« waren

60 Erich Kulke: Um das Erbe in der bäuerlichen Baukunst, in: Nationalsozialistische Monatshefte, 1939, Nr. 10, S. 21-32.

61 Kulke: Erbe (Anm. 60), S. 25.

62 Ebd., S. 25.

63 Ebd., S. $25 \mathrm{f}$.

64 Ebd., S. 29.

65 Ebd., S. 30.

66 Ebd., S. 31. - Lixfeld: Aufbau (Anm. 55), S. 212.

67 Erich Kulke: Das schöne Dorf. Eine Anleitung für die Gestaltung des deutschen Dorfes, hg. vom Verwaltungsamt des Reichsbauernführers, Reichshauptabteilung I, Berlin 1937. In dem Buch setzt Kulke gute und schlechte Beispiele in Bildbeispielen gegenüber. - Entsprechendes gilt für die von Kulke bearbeitete »Land-Baufibel«, München [1943]. 
Schwerpunkte Kulkes publizistischer Tätigkeit; Themen, die ihre Wurzeln auch in der Jugendbewegung hatten. So wurde schon in der Zeitschrift Wandervogel 1911 auf "Augenbeleidigungen " an Scheunen in Form von Plakaten hingewiesen und damit die am Ende des Kaiserreichs aufkommende Zivilisationskritik sichtbar. $^{68}$

Kulke hatte bei seinen Plänen stets die Verhinderung der Landflucht im Sinn, der er mit arbeits- und betriebswirtschaftlichen Aspekten bei »heimatgebundenen « Neubauten begegnen wollte, die letztlich zu einer Leistungs- beziehungsweise Ertragssteigerung führen und somit die Autarkie oder wie es damals hieß die "Ernährungssicherung", vor allem im Hinblick auf den möglichen Krieg, herbeiführen sollten. 1938 bearbeitete Kulke gemeinsam mit dem Architekten, Heimatschützer und frühen Nationalsozialisten Werner Lindner (1883-1964) u. a. den Band »Das Dorf. Seine Pflege und Gestaltung«, in dem er u. a. über »Das neue, heimatgebundene Bauen« schreibt, wobei er die gesetzlich am 14. Juli 1933 fixierte, durch das Reich übernommene Schaffung von neuen Höfen mit dem Ziel einer "Neubildung des deutschen Bauerntums" und einer »Wiedergesundung des ländlichen Bauwesens « erwähnt. ${ }^{69}$ In diesem Kontext entwickelt er das "Wunschbild eines nationalsozialistischen Siedlerdorfes», dessen Modell er abbildete und ausführlich beschrieb. Es war in Teilen eine verkleinerte Ausgabe des städtischen Monumentalstils. ${ }^{70}$ Ein besonderes $\mathrm{Au}$ genmerk lenkt Kulke auf Anlagen des Gemeinwesens, so auf den Dorfanger als Festplatz der Dorfgemeinschaft und als Zentrum dörflichen Lebens. Im Dorfteich sollte sich die Feierhalle spiegeln, die als geistiger und kultureller Mittelpunkt der Gesamtanlage gedacht war und offenbar die Kirche ersetzen sollte. ${ }^{71}$ Ein weiterer Aspekt galt der »artgemäßen bäuerlichen Wohngestaltung « unter Einbindung des Dorfhandwerks, auch um Werte der »bäuerlichen Baukultur» wieder herzustellen. ${ }^{72}$

Das Handwerk stand neben eher auf das Brauchtum ausgerichteten Themen auch im Fokus der 1937 und 1938 von Erich Kulke herausgegebenen Reihe »Von deutscher Art und Kunst« mit »Bildmaterial zu volkskundlichen Arbeiten, die heute jeden deutschen Menschen angehen«. Der Reihentitel des sechs Bände umfassenden Mappenwerks galt Werten, »in denen deutsches Wesen und deutsche Lebensgesinnung Ausdruck und Gestalt gefunden haben. ${ }^{73}$ Mit kur-

68 Wandervogel, 1911, Nr. 6, S. 193 f., hier S. 193.

69 Erich Kulke: Haus und Hof des deutschen Bauern, in: Werner Lindner, Erich Kulke, Franz Gutsmiedl (Bearb.): Das Dorf. Seine Pflege und Gestaltung, München 1938, S. 7-11. - Ders.: Das neue, heimatgebundene Bauen, in: ebd., S. 216-219, hier S. 219, Abb. 513-515.

70 Lixfeld: Aufbau (Anm. 55), S. 110.

71 Ebd., S. 212.

72 Kulke: Bauen (Anm. 69), S. 216.

73 Text im Klappendeckel zu Marie Adelheid Reuß zur Lippe: Deutscher Hausrat, Leipzig 
zen Textheften sollten die Mappen als Unterlagen für Vortragsabende dienen. Kulke selbst schrieb über die »Mahnmale des deutschen Heldentums «. ${ }^{74}$

Im November 1939 wurde Kulke vom Verein für Bauerntumskunde zum Leiter der Arbeitsgemeinschaft »Das bäuerliche Haus" bestimmt, konkret ging es um das Arbeitsgebiet «Das bäuerliche Haus als Heimstätte boden- und blutsgebundener Geschlechter unter der Odalsrune. Die Hausgemeinschaft und Heimgestaltung bäuerlichen Lebensstiles. ${ }^{75} 1940$ wurde der Verein umbenannt in »Gesellschaft der Freunde deutschen Bauerntums«. Nun galt ein besonderer Aspekt dem »lebensgesetzlichen Landbau«.

1941 veröffentlichte Kulke in der Zeitschrift »Odal« den Aufsatz »Deutsches Siedlungsgut im Osten ${ }^{76}{ }^{7}$ Ziel des Aufsatzes war es, den »deutschen Siedlungseinfluß, und zwar vornehmlich nach der Kulturtat leistungsfähiger deutscher Bauerngeschlechter « anhand von Siedlungsformen zu belegen. ${ }^{77}$ Zudem untermauerte der Autor auch die vom Regime gewollte Eroberung neuen Lebensraums im Osten, wenn er argumentierte, dass das Land dem Volk gehöre, welches in der Lage sei, eine höhere Kultur zu bringen, womit er Deutschland meinte. Hinsichtlich der Bodenbearbeitung sprach er von einem Kulturgefälle von Westen nach Osten, dass eben mit der Entfernung von Deutschland zusammenhing. ${ }^{78}$ Positiv hob Kulke jedoch den Kinderreichtum in Polen hervor, auch wenn er die Kinder als ungepflegt und arm beschrieb: „Urwüchsig und lebenskräftig wachsen sie heran, bevölkern die Dörfer und das Land und bewahrheiten damit die Tatsache, daß nicht nur die Höhe der Kultur allein, sondern vor allem die blutliche Lebenskraft und der Lebenswille den Bestand eines Volkes und den Besitz eines Landes sichern. Wir setzen zwar der Zahl die Güte und den Wert entgegen. Aber dennoch bleibt das unser Ziel [...]: den wiedergewonnenen ostdeutschen Lebensraum durch volkreiche Bauerndörfer mit

[1938]. - Die Autorin, von 1927 bis 1936 verheiratet mit dem Rassetheoretiker Friedrich Kurt gen. Hanno Konopath, war für Kulke vielleicht keine Unbekannte, berichtete sie doch über den Bundestag der Adler und Falken, war eine Förderin der Artamanen, hatte u. a. Traktate über »die naturgemäße Pflicht der snordischen Frau zur Mutterschaft und Aufzucht rartgerechter Kinder « publiziert und besaß enge Verbindungen zum Reichsbauernführer Darré sowie zu anderen Nationalsozialisten. Sie selbst blieb nach dem Krieg eine unbeirrbare Nationalsozialistin. - Dazu: Marie Adelheid Konopath: Neunter Bundestag der "Adler und Falken« in Koblenz, in: Die Sonne, 1930, Nr. 7, S. 425f. Frdl. Auskunft von Dr. Susanne Rappe-Weber, AdJb, A 227 Nr. 21. - Mogge: Balder (Anm. 15), bes. S. 56f. - NPD. Wahre Liebe, in: Der Spiegel, 1965, Nr. 37, S. 50 f. - Lionel Gossman: Brownshirt Princess. A Study of the »Nazi Conscience«, Cambridge 2009.

74 Erich Kulke: Mahnmale deutschen Heldentums, Leipzig [1937].

75 BArch, WI (BDC) 8200001691.

76 Erich Kulke: Deutsches Siedlungsgut im Osten, in: Odal. Monatszeitschrift für Blut und Boden, 1941, 10. Jg., S. 125-129.

77 Ebd., S. 125.

78 Ebd. 
kinderreichen Bauernsippen zu besetzen und zu halten. $"{ }^{79}$ Auch hier sollte die Neuansiedlung mit Deutschen eines Tages auf Neubauernhöfen erfolgen, die scheinbar an den traditionellen Baustil anknüpften, aber doch politische Zielsetzungen verfolgten.

Solche Untersuchungen standen im Kontext der »Aufgaben der Volkskunde bei der politischen Neuordnung Osteuropas«, die explizit die Bauernhausforschung Kulkes einbezogen. So sollte ihm ein Mitarbeiterstab zur Verfügung gestellt werden, "um den Einfluß deutscher Siedlungskultur im russischen Raum und im Baltikum zu untersuchen und die erforderlichen Planungen für die künftige deutsche Siedlung im Baltikum, für die er die unerläßlichen Grundlagen schaffen kann, auszuarbeiten. ${ }^{80}$ Noch zu Beginn des Jahres 1944 suchte Kulke nach jungen Leuten, die alte, einst von ausgewanderten Deutschen gebaute Bauernhäuser in Transnistrien aufmaßen, bevor das Kriegsgeschehen sie zerstöre. ${ }^{81}$

Im April 1944 wurde Kulke, der direkt dem Beauftragten des Führers für die Überwachung der gesamten geistigen und weltanschaulichen Schulung und Erziehung der NSDAP unterstellt war, zum Hauptgemeinschaftsleiter befördert. $^{82}$

\section{Neuanfang nach 1945 ?}

1945 geriet Erich Kulke in sowjetische Kriegsgefangenschaft, aus der er im November 1948 entlassen wurde. Der Entnazifizierungsausschuss stufte ihn im April 1949 als entlastet ein. ${ }^{83}$

Von 1949 bis 1950 war Kulke Baurat an der Staatsbauschule in Holzminden, seit 1951 Leiter der Bauabteilung der Landwirtschaftskammer Hannover. Der Architekt knüpfte also erfolgreich an seine frühere Tätigkeit an, worauf auch sein 1963 erschienenes Buch "Neuzeitliche Baugestaltung in der Landwirtschaft« hinweist. 1965 übernahm er bis zu seiner Pensionierung 1973 den Lehrstuhl "Landwirtschaftliche Baukunde» an der Hochschule Braunschweig, wobei ihm Werterhaltung und Wertsteigerung der ländlichen Umwelt ein zentrales Anliegen war. ${ }^{84}$ Wohnte die Familie zunächst bei Hannover, so zog man

79 Ebd., S. 129.

80 BArch, Kanzlei Rosenberg - NS 8/245, zit. nach: Freckmann: Hausforschung (Anm. 2 ), S. 174.

81 Ebd., S. 183.

82 Openjur (Anm. 30), Satz 14.

83 Ebd., Satz 17.

84 Reinhardt Guldager: Erich Kulke - Landbaumeister und Lehrer, in: Joachim Grube, Carl 
1968 in den »Schulzenhof « nach Bussau im Wendland, in einen Rundling. ${ }^{85}$ Diese Dorfform hatte Kulke wohl schon als Student ${ }^{86}$ kennengelernt und wiederholt in seinen Publikationen vor 1945 erwähnt. ${ }^{87} 1941$ vertrat er »die Ansicht, daß der im Runddorf vorherrschende Ordnungs- und Planungsgedanke deutsch-germanisch genannt werden muß. ${ }^{88}$ Doch heute weiß man, dass hier vorwiegend slawische Siedler lebten. Bereits vor Inkrafttreten eines entsprechenden Denkmalschutzgesetzes setzte sich Erich Kulke intensiv für den Erhalt der Rundlinge als Kulturgut ein, wofür er auch 1975 das Große Niedersächsische Verdienstkreuz erhielt. Zwischen 1976 und 1978 gab es in Bussau auch Älterentreffen des Wandervogels Deutscher Bund, und der Ort galt als eine Art Wallfahrtsort für Jugendbewegte. ${ }^{89}$

Die allgemeine Situation nach dem Krieg und die berufliche Einbindung ließen Kulke zunächst wenig Zeit, sich für den früheren Bund zu engagieren. Auf der Pfingsttagung des Kronacher Bundes 1954 auf dem Ludwigstein erkannte man die "Notwendigkeit des Weiterbestehens unserer Kameradschaft", denn »[d]ie Tatsache, daß wir uns nach mehr als 20 Jahren wieder zu einer Gemeinschaft zusammengefunden haben, ist uns ein eindeutiger Beweis von der Stärke der uns einst bewegenden Idee. ${ }^{90}$ Unter anderem sollte das Zusammengehörigkeitsgefühl gestärkt werden. Kulke umriss die Aufgabenstellung des Bundes für die Zukunft und betonte, »daß die einst aus der deutschbewußten Jugendbewegung gewachsenen Ziele niemals für die Erhaltung der Volkssubstanz notwendiger in ihrer Auswirkung sind als heute $[\ldots] \aleph^{91}$ Die vor dem Krieg erkannten Ziele wie Leistungswilligkeit, anständiger und sauberer Charakter, gesunder Körper, lebensstarke Familie und die Verankerung im deutschen Volkstum betrachtete er darin als »zeitlose unveräusserliche Werte«. Wesentlich war für ihn, dass die Mitglieder des Bundes mit ihren Kindern ein »deutsch-

Ingwer Johannsen: Eine Zukunft für das Bauen auf dem Lande. Festschrift für Erich Kulke, Münster-Hiltrup 1978, S. 15-22, hier S. 20.

85 August Quis: Nachruf Erich Kulke 1908-1997, in: Hannoversches Wendland, 2001, 15. Jahresheft 1994-1997, S. 329-331.

86 Erich Kulke: Heinrich Tessenow durchwandert das hannoversche Wendland, Bussau 1978.

871978 erhielt Erich Kulke für seine Verdienste die Tessenow Medaille in Gold der Alfred Toepfer Stiftung FVS.

88 Kulke: Siedlungsgut (Anm. 76), S. 127. - An anderer Stelle konnte man lesen, »daß die Erbauungspläne [der Rundlinge; C.S.] eine sinnvolle Eingliederung in den 4-, 6- oder 8fachen nordischen Horizontkalender erfuhren, daß ein solches Richtungsbild die Umsetzung des urgermanischen Sonnenjahres in die Landwirtschaft darstellte.« S. Erich Kulke: Die bäuerliche Siedlung, in: Wilhelm Hansen: Das deutsche Bauerntum. Seine Geschichte und Kultur, Berlin 1938, S. 149-212, hier S.165f.

89 Quis: Nachruf (Anm. 85), S. 331.

90 AdJb, P 1 Nr. 244 (Personenmappe Erich Kulke): Rundbrief Nr. 1 des Wandervogel Deutscher Bund vom 01.10.1954, S. 3.

91 Ebd., S. 4. 
betontes Leben « führten und dass das geistige Erbe des Dichters Georg Stammler (1872-1948) nicht verloren ginge. Der völkische Autor genoss in der Jugendbewegung besonders nach 1918 größtes Ansehen. Er war Mitglied der Adler und Falken sowie der Deutschen Glaubensgemeinschaft, seit 1923 im Vorstand der Deutschen Bauernhochschule und trat schließlich 1925 in die NSDAP ein. Er hatte sich zudem für die Bildung von Siedlergemeinschaften eingesetzt, in denen wirtschaftliche Arbeit und soziale Bindung zu Mitteln "geistiger Lebensführung « erhoben werden sollten. ${ }^{92} \mathrm{Zu}$ den tagesaktuellen Aufgaben gehörte laut Kulke die Unterstützung der Witwen gefallener Bundeskameraden mit ihren Familien, besonders in der DDR, der "Sowjetzone», wie er es nannte.

Nachdem sich Mitglieder des ehemaligen Bundes der Adler und Falken zum Dörnbergbund zusammengeschlossen hatten, nahm Kulke Ostern 1954 an einem Dörnbergtreffen teil. Er konstatierte zahlreiche Querverbindungen der beiden Älterenbünde, die ihre Wurzeln in der gemeinsamen Ausrichtung der früheren Bundesarbeit hatten. Diese lag wohl besonders in der einstigen gemeinsamen Beteiligung an der Deutschen Glaubensbewegung. ${ }^{93} 1955$ und 1956 kam es dann zu ersten Treffen des Wandervogels Deutscher Bund nach dem Krieg auf Burg Ludwigstein mit je rund 200 Teilnehmern. Auf dem Pfingsttreffen 1957 wurde die Eingliederung der Mitglieder des Älterenkreises Wandervogel Deutscher Bund in der »Vereinigung Jugendburg Ludwigstein « beschlossen. ${ }^{94}$

Zwischen 1959 und 1963 war Erich Kulke Vorsitzender der »Vereinigung Jugendburg Ludwigstein «. In diesen Jahren ging es verstärkt um Bau-, Planungsund Finanzierungsaufgaben im Zusammenhang mit dem am 11. Oktober 1963 eröffneten Meißnerbau, ${ }^{95}$ weshalb seine Wahl in dieses Amt gut nachvollziehbar ist. Der von dem Architekten Jürgen Jaeckel (gest. 1962) geplante Bau sollte vor allem eine Bleibe für das Archiv der deutschen Jugendbewegung werden.

1961 und 1962 richtete Kulke auf der Burg »Ost-West-Wochen« aus, die Probleme der deutschen Teilung und einer möglichen Wiedervereinigung thematisierten. ${ }^{96}$ Als Erich Kulke dann 1979 über die Geschichte des Bundes re-

92 Justus H. Ulbricht: Völkische Erwachsenenbildung. Intentionen, Programme und Institutionen zwischen Jahrhundertwende und Weimarer Republik, in: Puschner: Handbuch (Anm. 18), S. 252-276, bes. S. 270-272. - Breuer, Schmidt: Kommenden (Anm. 5), S. 417 f.

93 Mogge: Balder (Anm. 15), S. 51, 61.

94 Kulke: Weg (Anm. 14), S. 7. - 1. Freundesbrief vom 17.08.1957, unterzeichnet von Erich Kulke (AdJb, Z/300-2809).

95 Erich Kulke: Chronik des Meißnerbaues, in: Zur Einweihung des Meißnerbaues der Jugendburg Ludwigstein. Sonderheft der Ludwigsteiner Blätter. Witzenhausen, am 11. Oktober 1963, S. 11-23. Die Eröffnung des Baus war für Kulke zugleich das Ende der aktiven Burgarbeit.

96 Gideon Botsch: Zwischen Tradition und Rezeption. Völkische Jugendbünde und nationalistische Jugendverbände in der Bundesrepublik, Manuskript, im Druck. 


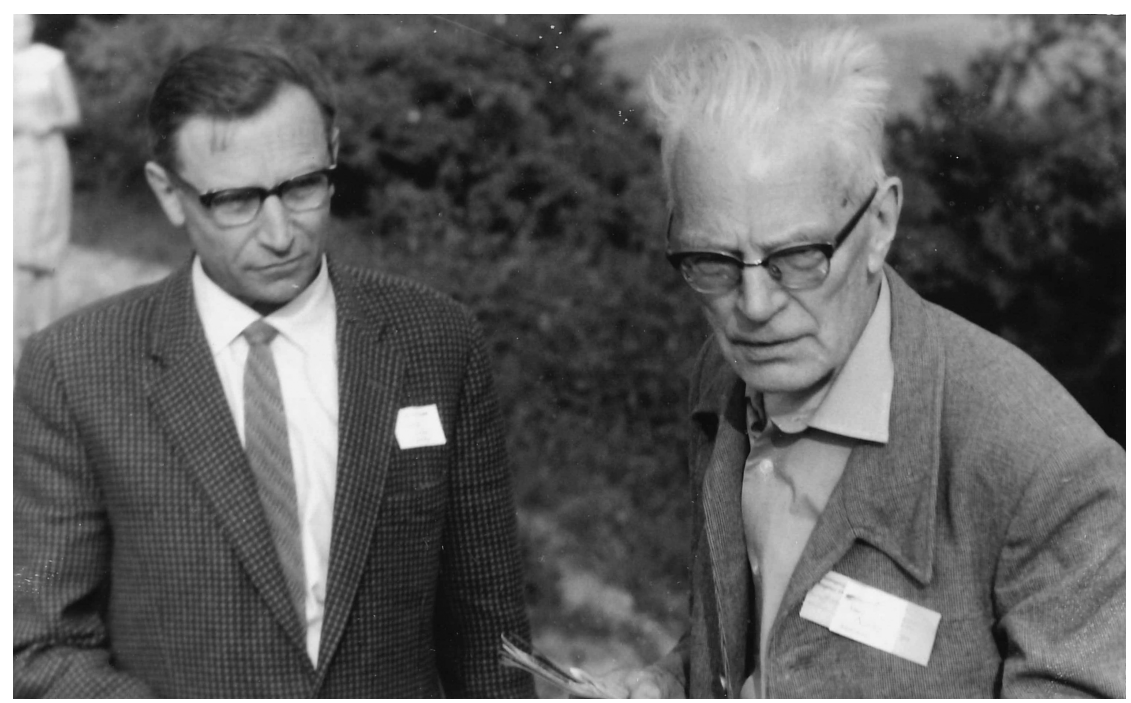

Abb. 21: Zwischen 1959 und 1963 war Erich Kulke (links) Vorsitzender der Vereinigung Jugendburg Ludwigstein.

flektierte, erwähnte er den »Irrweg von 1933 bis 1945« und sah die Notwendigkeit der Selbstkritik und Selbstbesinnung, der Veränderung: «Unser Blickfeld hat sich für ein europäisches Denken und eine humane Weltoffenheit geweitet, und wir gestehen uns, daß unsere vordergründig nur volkbezogene Einstellung begrenzt und einseitig war und Gefahren in sich barg. Das alles [...] schmälert aber nicht das ehrliche Ringen von damals, als unsere Liebe, unser Herz Deutschland galt - aber, das wollen wir uns sagen, einem Deutschland, frei von jeglichem Rassenhaß und nationalem Fanatismus. ${ }^{97}$ Die hier angedeutete Öffnung zur Welt, die Kulkes Ausführungen verschiedentlich zu entnehmen ist, drückte sich auch 1979 im Setzen des »Bussauer Heimatsteins" aus, der als »Erinnerungstafel für unsere verlorenen Gebiete Ostdeutschlands« gedacht war. »Er berührt damit das Verhältnis des deutschen Volkes zu seinen östlichen Nachbarn und dient dem Bemühen, in Ausgleich und Versöhnung für unsere Völker im östlichen Europa in eine bessere Zukunft zu finden. ${ }^{98}$

Erich Kulke war zudem Mitglied des 1950 gegründeten "Arbeitskreis für Hausforschung». 1982 erschien der Aufsatz »Hausforschung im Dritten Reich» von Klaus Freckmann, in dem dieser unter anderem auf die scheinbar wissenschaftlich fundierte Verbindung Rasse - Volkstum - Bauerntum - Hausform und die Funktion Kulkes in der "Mittelstelle deutscher Bauernhof« zur Zeit des

97 Kulke: Weg (Anm. 14), S. 3.

98 Erich Kulke: Gedanken um den Bussauer Heimatstein. Bussau 1979 (AdJb, Personenmappe Kulke). 
Nationalsozialismus hinwies. Die Äußerungen stießen teilweise auf heftige Kritik innerhalb des Arbeitskreises. ${ }^{99}$ Kulke forderte Freckmanns Austritt, doch am Ende bewirkte der Aufsatz das Gegenteil: den Austritt Kulkes aus dem Arbeitskreis. $^{100}$

\section{Fazit}

War die Einordnung der Person Erich Kulkes für ein Gericht in Frankfurt an der Oder 2008 relativ eindeutig, so ist sie in historischer Perspektive deutlich komplexer. In einem wiederaufgenommenen Prozess, in dem Kulkes Erben die Ausgleichszahlung für ein Grundstück in der DDR forderten, kam das Gericht in der abgewiesenen Klage zu dem Schluss, dass der Erblasser »dem nationalsozialistischen System [...] erheblichen Vorschub geleistet « habe durch die Propaganda in der Schrift »Hin zum Tage der deutschen Freiheit!«, die dieser zusammengestellt und verbreitet hat. ${ }^{101}$ Zudem hatte das Gericht »keinen Zweifel daran, dass der Erblasser bei seinem vielfältigen Engagement wesentlich und willentlich für das nationalsozialistische System tätig geworden ist. « ${ }^{102}$

Der Wille oder die Bereitschaft für den Nationalsozialismus zu arbeiten, wurde bei Kulke in der Jugendbewegung gelegt, wo nicht nur er auf der Suche nach einer neuen Weltanschauung und neuen Lebensinhalten war. Die Blut- und Bodenideologie Walther Darrés war dem völkisch orientierten Erich Kulke geläufig, er formulierte ähnliche Gedanken und leistete mit seinen zahlreichen Aufsätzen in vielerlei Hinsicht sogar Vorarbeiten für die völkisch-rassistisch bestimmte NS-Bauerntumsideologie. Sicher glaubte er, im Rahmen der NSOrganisationen seine Forschungen und in bündischer Zeit entwickelten Ideale realisieren zu können. Vor allem bot sich dem jungen Familienvater in der »Mittelstelle deutscher Bauernhof « bzw. in der »Reichsarbeitsgemeinschaft für Volkskunde« die Möglichkeit, sein Wissen anzuwenden und sich als Nationalsozialist zu profilieren. ${ }^{103}$ Ferner konnte er zahlreiche Veröffentlichungen - besonders in den Organen des Amtes Rosenberg - publizieren, die die nationalsozialistische Ideologie unterstützten. In seinem Arbeitsumfeld fanden sich

99 Klaus Freckmann: 50 Jahre Arbeitskreis für Hausforschung, S. 2f., http://www.arbeits kreisfuerhausforschung.de/files/Freckmann_50 Jahre_AHF.pdf [09.06.2014].

100 G. Ulrich Großmann: Völkisch und national - Der »Beitrag« der Hausforschung. Wiederaufleben der Runenkunde des SS-Ahnenerbes, in: Uwe Puschner, G. Ulrich Großmann (Hg.): Völkisch und national. Zur Aktualität alter Denkmuster im 21. Jahrhundert, Darmstadt 2009, S. 31-64, hier S. 36. - Ferner mdl. Auskunft von Klaus Freckmann im September 2014.

101 Openjur (Anm. 30), Sätze 46, 60.

102 Ebd., Satz 73.

103 Gajek: Feiergestaltung (Anm. 57). 
einige Mitstreiter aus der Jugendbewegung, zumeist aus dem Bund der »Adler und Falken« (Matthes Ziegler, Hans Strobel) - mithin gab es Netzwerke.

Nach dem Krieg gelang es Kulke auf viele seiner Arbeiten aus dem Architekturbereich und der Denkmalpflege aufzubauen und er machte wie einige andere Bündische Karriere an einer westdeutschen Hochschule. Die »Kameraden« aus seinem alten Bund blieben für ihn lebenslang wichtig, das Adjektiv "deutsch" eine entscheidende Vokabel, das »deutschbetonte Leben« ein verinnerlichtes Leitmotiv, doch auch die Versöhnung mit Polen rückte in den Fokus. So zeigte er sich als typischer Vertreter seiner Generation. Das bündische Organisationstalent, die Freude an der Gruppenarbeit konnte er ebenso wie sein Fachwissen - befreit von völkischen Momenten - bei seinen neuen Aufgaben im Nachkriegsdeutschland gut einbringen. 